\title{
Detection of Strong Shadows in Monochromatic Video Streams
}

\author{
Christian Bräuer-Burchardt \\ Friedrich Schiller University Jena, Dept. of Computer Science, \\ Digital Image Processing Group, D-07740 Jena, Germany \\ delphin@minet.uni-jena.de
}

\begin{abstract}
A new robust method to shadow detection applicable to moving object tracking tasks is presented. The information about existence and kind of shadows from the preceding images of the sequence is used to predict the properties of the shadows in the current image. Shadow points are identified by comparison to the expected shaded grey value using the shading function. The technique allows mainly the detection of strong shadows from direct sunlight.
\end{abstract}

\section{Introduction}

The detection of shadows of moving objects is a typical problem of image processing. Whereas in recent years powerful techniques using colour image sequences have been established, more difficulties arise in the case of monochromatic images $[2,6,8,10]$. However, colour images have their disadvantages, too. There are many reasons to use monochromatic images, e.g. less data to store and to process. In fact, our problem is the shadow detection in monochromatic image sequences.

The detection and tracking of moving objects in video sequences may be considered as the background problem of our project [1, 4, 9]. Usually walking people or moving vehicles are to be considered. If there is the task to count objects or to analyse their shape, shadows may disturb the identification and quantification becomes incorrect.

Depending on the illumination conditions the objects to be detected have shadows. These shadows may be hardly noticeable on the one hand or very strong, leading to total eclipse of the mapped area on the other hand. Thus a general shadow concept should enclose weak shadows as well as strong shadows and the whole range between.

Our situation can be described as follows. Given an image sequence as a file or a real-time process. A background image should be present or its calculation should be possible, e.g. by median filtering. The moving objects in the current image are detected by image subtraction with the background image. Some morphological procedures follow in order to detect object points with similar grey values to the background. However, the subtraction procedure usually leads to the classification of shadow points as object points. Object enlargement, object fusion, or detection of more objects than present may occur, and thus quantification errors arise. The final goal should be the detection of the shadow points in order to get a true quantification of the objects.

See fig. 1 for illustration of a typical situation. In the left image there are objects with shadows, in the middle the background image (including permanent shadows) is shown, and the right is the difference image. 


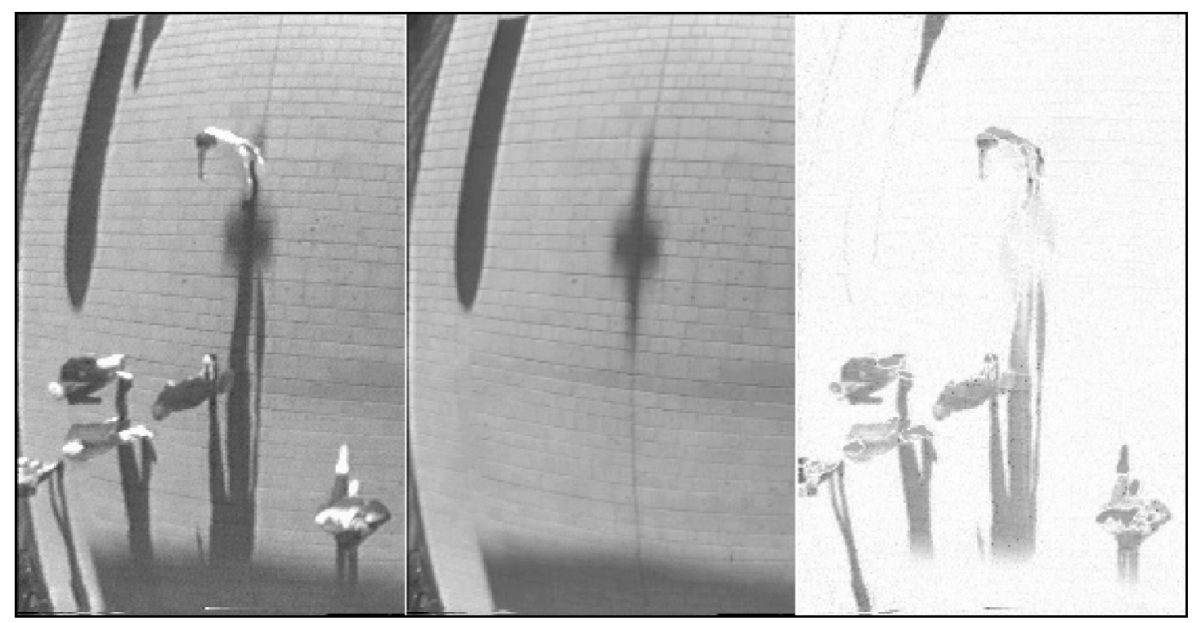

Fig.1. Persons with shadows (left), background image with permanent shadows (middle), image difference (right)

There are a number of recent publications concerning shadow detection. Works analysing colour image sequences predominate in the literature $[3,5]$. Unfortunately, the majority of these methods is not applicable to our situation because of the absence of colour information. Algorithms using colour information are usually based on the effect, that that shadows influence luminance of the image as well as colour intensity, but not the colour itself. The more difficulties in monochromatic shadow detection are reflected in fewer works considering grey image sequences.

An interesting shadow detection technique using monochromatic image sequences is presented by Stauder [10]. Shadows are classified as umbra (not illuminated) and penumbra (diffusely illuminated area in the shadow of an object). The presented algorithm is based on the heuristic combination of three features, each having two or three possible states. The three features are illumination change (with states unchanged and changed), edges (with states no edge, static edge, and dynamic edge), and shading (with states no result, unique, not unique). A rule for point classification is stated for each of the 18 possible combinations. Information about the neighbourhood of a point is additionally used in four of the 18 cases.

Onoguchi [6] uses an approach based on inverse perspective mapping. There the assumption is used that an object covers its shadow by projection on the background. This method needs additional information about the orientation of the luminance. Jiang [2] presents a shadow detections method based on shadow intensity and geometry starting in dark regions. Prati [7] gives an survey over several shadow detection techniques together with a detailed comparison and an experimental evaluation of four methodologies including the determination of error rates using different standard video sequences. 


\section{Shadow description}

Stauder [10] suggests to describe the luminance $s(x, y)$ of the image points as the product of irradiation $E(x, y)$ and reflection $\rho(x, y)$. The irradiation $E(x, y)$ is obtained according

$$
E(x, y)= \begin{cases}c_{A}+c_{P} \cos (N(x, y), L) & ; \text { if illuminated } \\ c_{A}+k(x, y) c_{P} \cos (N(x, y), L) & ; \text { if penumbra } \\ c_{A} & ; \text { if shaded }\end{cases}
$$

where $c_{P}$ and $c_{A}$ represent the intensities of light source and ambient light, $L$ the direction of illumination, and $N(x, y)$ the surface normal. Penumbra means a partly illuminated area but this case needs extra consideration and will be considered later. Forming the difference $\operatorname{diff}(x, y)$ of the luminance $s(x, y)$ between an illuminated point $(x, y)$ and the same point occluded by a shadow we get

$$
\operatorname{diff}(x, y)=\rho(x, y) c_{p} \cos (N(x, y), L) .
$$

Now we assume that the background covered by one connected shadow is almost plane. Thus we can guess a constant value for $c_{p} \cos (N(x, y), L)$ and hence $\operatorname{diff}(x, y)$ is proportional to $\rho(x, y)$. The representation of the illumination difference $\operatorname{diff}(x, y)$ is the difference of the grey values between the current image with shaded points $(x, y)$ and the illuminated background image.

Let $G$ the current image with grey values $g_{c}(x, y)$ at pixel position $(x, y), B$ the background image with $g_{b}(x, y)$ and $D$ the difference image with $d(x, y)$, respectively. Note that if the range of $G$ and $B$ is the interval $[0,255]$, the range of $D$ is $[-255,255]$.

As empirical shading difference function (SDF) the grey value difference of a shaded point $p=(x, y)$ to the corresponding unshaded point in the background image should be regarded:

$$
s d f(x, y)=g_{c}(x, y)-g_{b}(x, y) .
$$

Note that $p=(x, y)$ should has been classified as a shaded point and that grey value 255 means black and zero means white. Now the general shading function $s f$ should be defined as the expected grey value difference depending on the grey value in the background image and its co-ordinates: $s f(x, y)=E(s d f(x, y))$. Depending on the properties of the used camera a monotone function for $s f$ is expected. This function is linear in the ideal case, eventually disturbed by the so called gamma-function. Thus in the first step the curvature of the monotone function $s f$ should be analysed. If it is not linear, a correction function $c f$ should be calculated to transform it into a linear one which should be used for the shadow detection. 
Let the function $s f$ linear without loss of generality: $s f\left(g_{b}\right)=a g_{b}+b$, where $g_{b}$ is the grey value in the background image. Because it should hold $s f\left(g_{b}\right) \geq 0$ and $g_{1}<g_{2} \rightarrow s f\left(g_{1}\right) \geq s f\left(g_{2}\right)$ in the whole interval [0, 255], it follows: $-1 \leq a \leq 0$. In the extreme case of no diffuse light $a$ will be close to -1 and all shadow points have almost the same black grey value. On the other hand, if there is only diffuse light, $a$ will be close to zero and the shadows become weak.

Additionally, $s f$ may be regarded depending on the position in the image due to different illumination conditions: $s f=f(g, x, y)$. That means the straight line parameter $a$ and $b$ are depending on the image co-ordinates and thus $a=f(x, y)$ and $b=f(x, y)$. Finally, the shading function may be expressed as:

$$
s f\left(x, y, g_{b}(x, y)\right)=a(x, y) g_{b}(x, y)+b(x, y) .
$$

The grey value difference of shaded area depending on the grey value of the illuminated background image without any shadow is illustrated in fig. 2 .

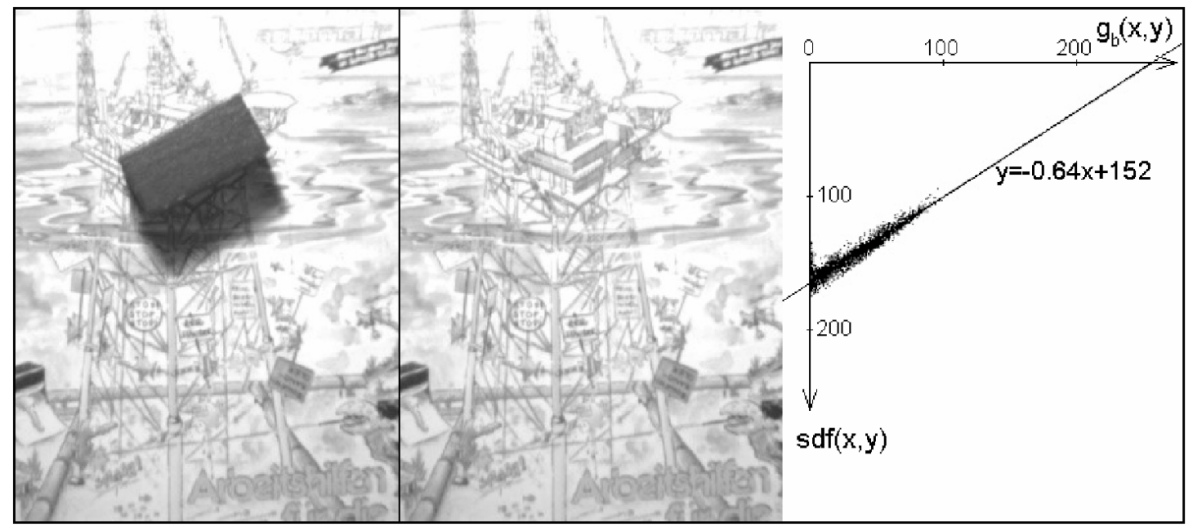

Fig.2. Object with shadow (left), background image (middle), grey value difference depending on grey value in the background image and regression line (right)

\section{Shadow detection algorithm}

\subsection{Determination of the shading function}

Assuming all shadow points has been determined in the preceding image. For all these points the grey value difference between the current and the background image is considered as a function of the background grey value: $s d f(x, y)=f\left(g_{b}(x, y)\right)$. In that case $s d f(x, y)=d(x, y)$. The detected shadow points are regarded as a sample of observations and the functional dependence should be determined. As already mentioned before, the function $s d f$ is assumed to be linear. This shall be checked by regression 
analysis. Otherwise a transformation into a linear function according to a correction function $c f$ should be realised.

Now $s f$ is calculated by linear regression to the values of $s d f(x, y)$. The expression of a straight line with only two parameters $a$ and $b$ may be chosen, because the case of a vertical straight line can be excluded. A shadow point $(x, y)$ has the expectation value in the difference image: $s f(x, y)=a g_{b}(x, y)+b$.

Eventually, as already mentioned, local differences due to varying illumination conditions or different normal vectors of the surfaces may occur. This can be checked by comparing the line coefficients $a_{i}$, and $b_{i}(i=1, \ldots, m)$ obtained from shadows at $m$ different positions $p_{i}=\left(x_{i}, y_{i}\right)$ in the image. That means the coefficients obtained at the same position have to be used. If there was no shadow at the current location before, the actual value of $s f$ at a certain position $(x, y)$ may be obtained by inter- or extrapolation according to the different distances between the actual point location and the recent shadow positions. However, if the normal vectors of the several surfaces are different, this kind of processing may be faulty.

See for example fig.3. Here four shadow regions are extracted and the values for $s d f(x, y)$ are shown in dependence on $g_{b}(x, y)$ together with the linear regression lines representing $s f(x, y)$ in the four regions. Note that diffuse light is absent and $a_{i}$ is almost -1 in all four cases.

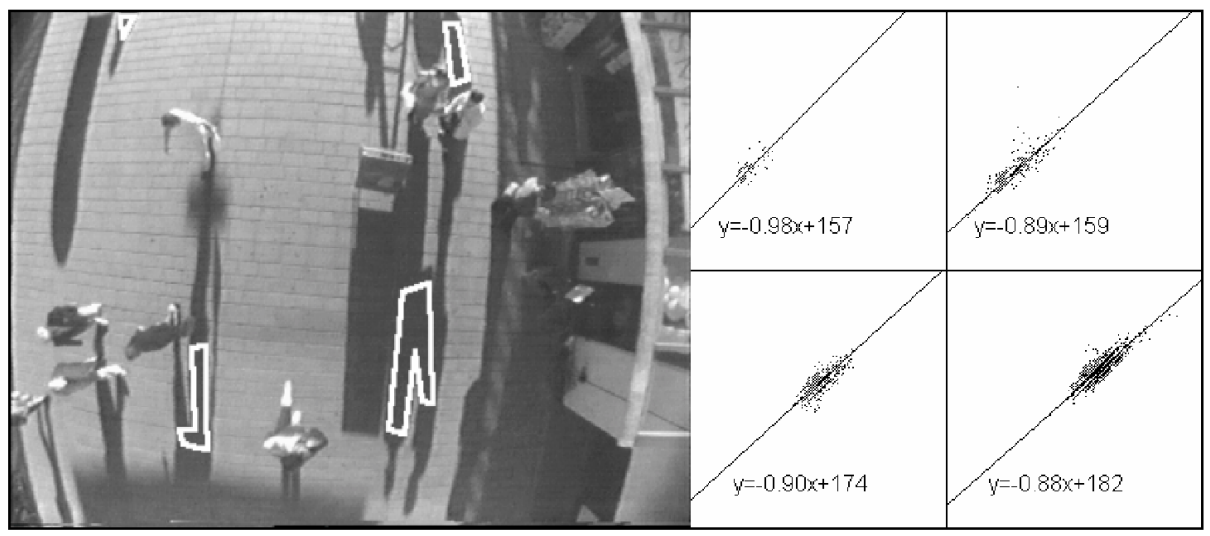

Fig.3. Four shadow regions (left) with corresponding values of $s d f(x, y)$ vs. $g_{b}(x, y)$ with regression lines (right)

\subsection{Weak shadow detection and processing}

In contrast to the strong shadows originated by direct illumination weak shadow may occur caused by diffuse illumination in indoor environments. Considering such images the differences between the shaded area and the background image are small and subjectively often not recognised. Nevertheless differences are present and lead to the detection of object point candidates.

See the example in fig.4. As we see almost no difference between the image and the background in the area marked by the contour, the $s d f$ is not zero. However, the correlation of the regression line to the points is weak because the empirical shading 
function is far from a straight line. Thus the determined equation for the regression line is uncertain. In such cases the shading function could be set to a constant value instead of the regression line equation, e.g. to the mean difference value: $s f(x, y)=\frac{1}{n} \sum_{1=1}^{n} g\left(x_{i}, y_{i}\right)=$ const $=b$ over all $n$ shaded points $(x, y)_{i}$. However, this is not an adequate description of the weak shadow, because the shading function does actually not depend on the grey values of the background image.

If the shading function is set to a vertical line the constant $b$ indicates the expected difference between the grey values of the shaded image points and the background. All pixels with $|s d f-b| \leq t h r$, where $t h r$ is a suitable threshold are candidates to be weak shadow points. However, the error rate is usually high in this case. That's why additional processing should be performed, e.g. similarity analysis as described in [1].

Penumbra can be considered as a special case of a weak shadow and should not be closer inspected here.

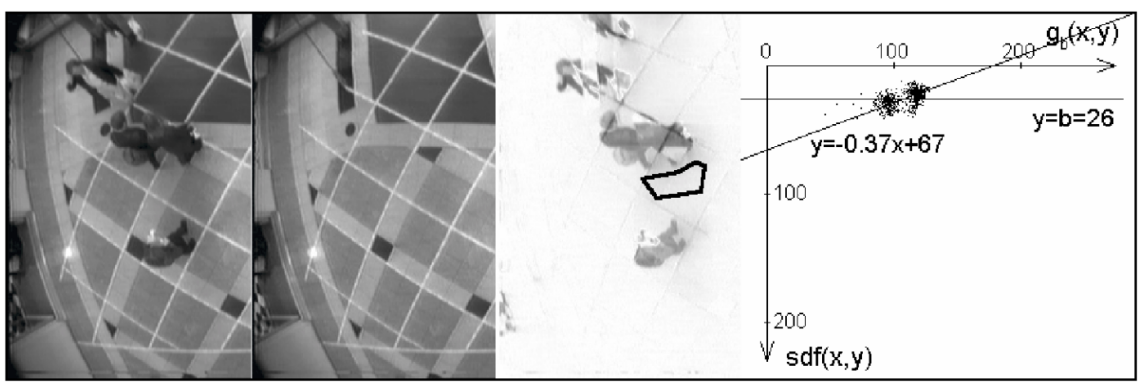

Fig.4. Image with weak shadows, corresponding background image, difference image with analysed weak shadow region, and values of $s d f(x, y)$ vs. $g_{b}(x, y)$ with regression line and constant approximation of $s f$ (from left to right)

\subsection{Shading point detection}

The estimation of the shading points can be described as follows. First, it is detected whether strong or weak shadows are present. All points obtained by the object detection procedure are candidates (SPC) to be shadow points. After shading point detection a point is classified as a proper object point or shadow point, respectively.

If strong shadows are present, for all SPC the shading function $s f(x, y)$ is determined. The current value of $g_{c}(x, y)$ is compared with $s f(x, y)$. If $\left|g_{c}(x, y)-g_{b}(x, y)-s f(x, y)\right|>v t h r$, the point is classified as a proper object point (no shadow). The value for the threshold $v t h r$ is obtained by variance analysis of the empirical shading function $s d f$ concerning the calculated shading function $s f$. The simple or double standard deviation seems to be a meaningful value for $v t h r$.

See the example of strong shadow detection as illustrated in fig.5. A morphological post-processing is applied to the detected shadow points in order to get connected regions of shaded and illuminated areas, respectively. 


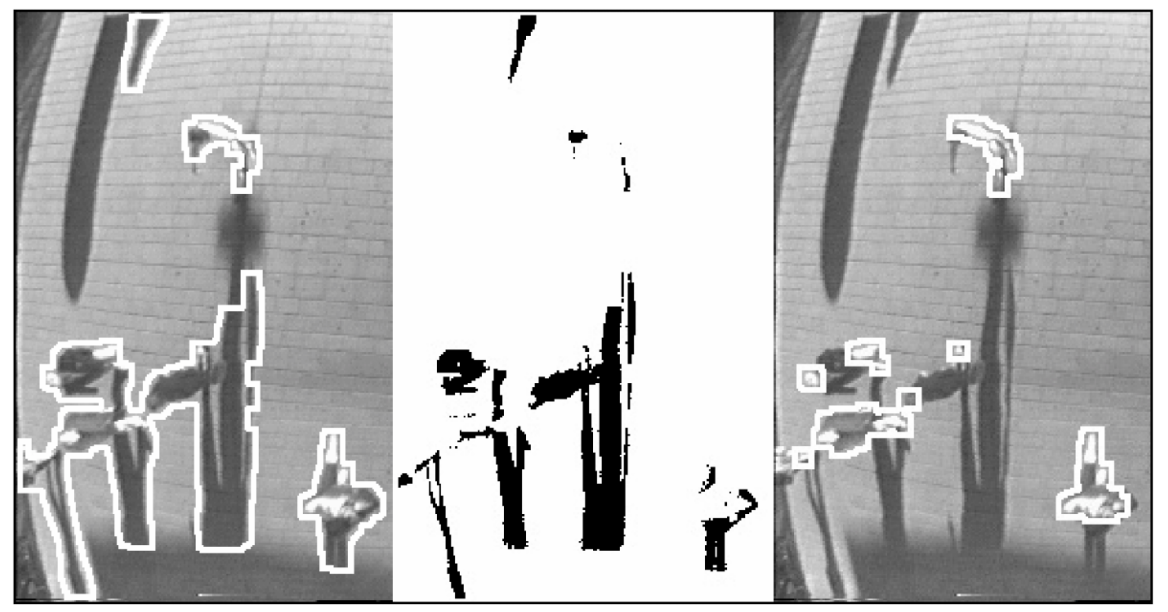

Fig.5. Original image with detected objects (left), detected shadows (middle), objects after shadow elimination

\subsection{Initial shadow analysis}

The first image of the sequence has no preceding frame where shadow could be analysed. Additionally, if there is no moving object in the image, there may be only illuminated area without any shaded region. If this happens for a long time, the illumination conditions might be changed, and it may be necessary to perform an initial shadow analysis in order to determinate the shading function.

In principle, two approaches are possible. First, some representative shaded regions are selected interactively by the user. The extracted $s d f$ values are the input for the shading function determination.

The second one works automatically, but the user must evaluate the results. The whole region of the detected objects is the input for the determination of the $s d f$. Only points with $d(x, y)>t h r$ are regarded considering just dark shadow points using a suitable threshold $t h r$. If the shaded area is small then the chances to extract the $s d f$ are small. However, if the shadow occupies the half or more of the region of potential shadow points, the regression line will be found exactly even if there are points disturbing the regression line determination.

\section{Summary, discussion, and outlook}

A new methodology of shadow detection in monochromatic video streams was presented. The main novelty is the approximation of the grey value difference between a certain shaded image point and the same not shaded point (in the background image) by a linear function depending on the grey values of the not shaded background image points. This function is obtained by linear regression of the detected shadow points of the preceding image of the sequence. 
A number of video streams with walking persons was analysed in order to count and track the pedestrians. In all sequences the shadows could be successfully detected and eliminated. The error rate of the counting process could be drastically reduced.

The method is not restricted in its application to person shadows. It may be used for elimination of vehicle shadows, too.

The main disadvantage of this method is the false classification of object points with the same grey value difference as the shading function as shadow points. In principle, this is not avoidable. However, these errors may be considered as statistically neglectable in an object determination and counting process.

A second disadvantage is the fact that in penumbra area the coefficients of the shading function are not constant. In practice, penumbra points are classified either as shadow points or as background points. This may lead to neglectable errors in the object detection task, but it should be avoided trying to extract the shape of the shadow more precisely. This problem should be in focus in the further research. In order to evaluate the presented technique comparing experiments to other methods necessary in the future work.

\section{References}

1. Bräuer-Burchardt, C.: Pedestrian Identification, Tracking, and Counting with Shadow Detection in Monochromatic Video Streams. Proc. 22 ${ }^{\text {nd }}$ IASTED Int. Conf. Modelling, Identification, and Control, 2003, 506-511

2. Jiang, C., and Ward, M.O.: Shadow segmentation and classification in a constrained environment, CVGIP - Image Understanding, 59(2), 1994, 213-225

3. Kaewtrakulpong, P., and Bowden, R.: An improved adaptive background mixture model for real-time tracking with shadow detection. Proc $2^{\text {nd }}$ Europ. Workshop on Advanced Video-based Surveillance Systems (2001), 149-158

4. McKenna, S.J., Jabri, S., Duric, Z., Rosenfeld, A., and Wechsler, H.: Tracking groups of people, Computer Vision, Graphics, and Image Processing, 80, 2000, 42-56

5. Mikic, I., Cosman, P.C., Kogut, G.T., and Trivedi, M.M.: Moving shadow and object detection in traffic scenes. Proc ICPR 2000, vol. I, (2000), 321-324

6. Onoguchi, K.: Shadow elimination technique for moving object detection. Proc ICPR'98, vol.I, 583-587, 1998

7. Prati, A., Cuicchara, R., Mikic, I., Trivedi, M.: Analysis and detection of shadows in video streams: a comparative evaluation. Proc IEEE-CS Computer Vision and Pattern Recognition conference (CVPR 2001), Kauai, Hawaii, USA, 2001

8. Sexton, G.G., Zhang, X.: Supression of shadows for improved object discrimination. IEEE Colloquium on Image Processing for Transport Applications, London, (1993), 9/1- 9/6

9. G. Sexton, X. Zhang, G. Redpath, and D. Greaves: Advances in automated pedestrian counting, Proc. IEE Int. Conf. on Efficiency, Costs, Optimization, Simulation and Environmental Impact of Energy Systems, 1995, 106-110

10. Stauder, J., Mech, R., and Ostermann, J.: Detection of moving cast shadows for object segmentation. IEEE Trans on Multimedia, 1(1): 65-76 